\title{
Variability in the management of line-related upper extremity deep vein thrombosis
}

\author{
Rafael Cires-Drouet ${ }^{1,2}$, Jashank Sharma ${ }^{1}$, Tara McDonald ${ }^{3}$, John D Sorkin ${ }^{3,4}$, Brajesh K \\ Lal $^{1,2}$ \\ ${ }^{1}$ Department of Vascular Surgery, University of Maryland School of Medicine, Baltimore, MD, USA \\ ${ }^{2}$ Vascular Service, Veterans Affairs Medical Center, Baltimore, MD, USA \\ ${ }^{3}$ Claude D. Pepper Older Americans Independence Center, University of Maryland School of \\ Medicine, Baltimore, MD, USA \\ ${ }^{4}$ Baltimore VA Medical Center Geriatric Research, Education, and Clinical Center, Baltimore \\ Veterans Affairs Medical Center, Baltimore, MD, USA
}

\begin{abstract}
Objectives: Central-venous devices are risk-factors for upper extremity deep vein thrombosis. We surveyed physicians to identify practice-patterns and adherence to American College of Chest Physicians guidelines.
\end{abstract}

Methods: The 13-question survey obtained physician-demographics and treatment-choices. Respondents were grouped into surgical and medical specialists. Data were reported as ratios and percentages, and compared using Fisher's exact test.

Results: We received 143 responses from physicians; $65 \%$ treated one-to-two new cases/month. Most physicians (69.2\%) used anticoagulation; 36.4\% retained the catheter and 32.9\% removed it. Medical-specialists retained catheters more often than surgeons $(p=0.027)$. For recurrences, $84 \%$ repeated anticoagulation; $50.3 \%$ retained the catheter. A majority anticoagulated upper-extremity deep-vein thrombosis in long-term catheters for three months only (55.1\%). Direct oral anticoagulants were used frequently (43.6\%). Only $10 \%$ believed that existing guidelines were appropriate and only $2.8 \%$ followed all guidelines.

Conclusion: There is great variability in treatment-decisions for upper-extremity deep-vein thrombosis. The existing guidelines are considered inadequate and not followed by most physicians.

Article reuse guidelines: sagepub.com/journals-permissions

Corresponding author: Brajesh K Lal, University of Maryland Medical Center, 22 South Greene Street, S10B00, Baltimore, MD 21201, USA.blal@som.umaryland.edu.

Declaration of Conflicting Interests

The author(s) declared no potential conflicts of interest with respect to the research, authorship, and/or publication of this article.

Ethical approval

This study was reviewed and approved by the University of Maryland Institutional Review Board. 


\section{Keywords}

Deep vein thrombosis; anticoagulation; catheter; upper extremity; intravenous device

\section{Introduction}

Modern clinical practice has seen an increased use of central venous devices including shortand long-term central venous catheters, pacemakers and defibrillator wires, hemodialysis catheters and chemotherapy ports. The Centers for Disease Control and Prevention recommends preferential use of upper extremity central venous access over femoral vein access to minimize catheter-related nosocomial infections. ${ }^{1}$ Increased use of upper extremity central venous access has led to an increased incidence of upper extremity deep vein thrombosis (UE-DVT) among hospitalized patients. It affects at least $2.2 \%$ of patients admitted to an intensive care unit (ICU) ${ }^{2}$ and $6 \%$ of patients requiring long-term intravenous catheters $^{3}$; however, the number may be even higher since the condition is not screened for in all patients. There are few prospective studies to inform treatment or prevention of UEDVT in patients with central venous devices. ${ }^{3-5}$ Most available information is derived from retrospective chart reviews and treatment recommendations remain inconclusive. ${ }^{6-9}$ In the absence of definitive high-quality data, the 2012 guidelines for the management of UE-DVT from the American College of Chest Physicians (ACCP) were based on less than ideal evidence (grade $2 \mathrm{~B}$ or $2 \mathrm{C}$ ) and on extrapolations from studies on lower extremity DVT. ${ }^{10}$ The low quality of data may be the reason that the most recent ACCP guidelines from 2016 do not provide any recommendations on anticoagulation treatment or catheter management for catheter-related UE-DVT. ${ }^{11}$ Furthermore, little is known about practice-patterns among physicians who treat patients with UE-DVT. In order to better understand current strategies used to manage patients with UE-DVT, we designed a survey and distributed it to physicians across a broad spectrum of specialties.

\section{Methods}

We developed a web-based 13-question survey using SurveyMonkey® (San Mateo, California, USA). Our analysis followed established guidelines for reporting medical surveys. ${ }^{12}$ Links to the survey were e-mailed to physicians from specialties including Cardiology, Critical Care, Emergency Medicine, Hematology/Oncology, Internal Medicine, Interventional Radiology, Nephrology, Orthopedic Trauma, Vascular Medicine and Vascular Surgery from the University of Maryland Medical System, as well as other academic institutions in the country. Participation was voluntary, responses were kept anonymous and no economic or other incentives were offered. The survey started in December 2017 and closed in March 2018. Survey questions (Figure 1) were designed to obtain demographic characteristics of the physician participants, the frequency with which they encountered and treated patients for UE-DVT, whether intravenous devices (IVDs) were associated with UEDVT, the treatment strategies they used, their preferred anticoagulants, their approach to prevention, and their opinion on the only existing guidelines for this condition. ${ }^{10}$ The survey was designed as a series of multiple-choice questions with space for comments. One question requested a free text answer (Question 2). Data were analyzed using excel 
(Microsoft Corp., Redmond, Washington, USA) and MedCalc Software bvba (Ostend, Belgium). Measures of central tendency, ratios, and percentages are reported. The several sub-specialties were collapsed into two groups, surgery and medicine and results were compared using Fisher's exact test. A two-tailed $p$ value $₫ 0.05$ was taken as indicating significance. The Institutional Review Board at the University of Maryland approved the survey; all participants provided informed consent.

\section{Results}

A total of 1784 physicians were invited to participate. One hundred and forty-three participants completed the survey (8\%). The distribution of respondents among Medical specialists was: Critical Care (20\%), Vascular Medicine (15\%), Internal Medicine (11\%), Cardiology (10\%), Hematology (5.5\%), Emergency Medicine (5.5\%), Neurology (2\%), Nephrology (1\%), and among Surgical specialists was: Vascular Surgery (11\%), General Surgery (3.4\%), Trauma Surgery (2.7\%), Cardiac Surgery (2\%), Thoracic Surgery (0.6\%), and Neurosurgery $(0.6 \%)$. The mean ( \pm standard deviation, $\mathrm{SD}$ ) number of years in practice for the physicians was $11.7( \pm 9.48)$ years with a range of $1-40$ years. Sixty five percent of physicians reported encountering and treating one-to-two patients with UE-DVT per month and only one participant reported treating more than 10 per month. A majority of participants $(83 \%)$ encountered patients with UE-DVT primarily in an in-patient setting and rarely $(6 \%)$ as outpatients. Half of the physicians attributed IVDs to be the culprit for a majority ( $>75 \%$ ) of UE-DVTs. Only $2 \%$ found IVDs to be an infrequent cause $(<25 \%$ of their cases) of UE-DVT.

Most participants started patients with newly diagnosed IVD-related UE-DVT on anticoagulation. Some respondents combined anticoagulation with IVD-removal (32\%), while others did not (36\%) A substantial minority elected not to anticoagulate their patients, regardless of whether they removed the IVD (16\%) or not (2\%). The ACCP 2012 guidelines 10 for catheter-related UE-DVT recommend leaving the catheter in place if it is working and to anticoagulate for 12 weeks or for as long as the catheter is in place. Only one-third of our participants (36.3\%) followed this guideline, one-third (32.87\%) would remove the catheter and anticoagulate, and the remaining one-third would not start anticoagulation (18.8\%) or would use a different strategy (10.49\%). The mean number of years that physicians were in practice was no different across the above three groups. Treatment strategies differed between surgeons vs. medical doctors ( $p<0.03$, Fisher's Exact test) (Figure 2); $17 \%$ of surgeons and $45 \%$ of medical specialists would leave the IVD in place and start anticoagulation; $40 \%$ of surgeons and $30 \%$ of medical specialists would remove the catheter and start anticoagulation, while $25 \%$ of surgeons and $12 \%$ of medicine physicians would remove the catheter but not start anticoagulation. Direct oral anticoagulants (DOACS) were the anticoagulants of choice for $39.2 \%$ of respondents, unfractionated heparin (UFH) bridged with warfarin for 35\%, and low-molecular weight heparin (LMWH) monotherapy for 14\%. Antiplatelet therapy (aspirin or clopidogrel) was used for independent treatment for UE-DVT by $8.5 \%$. In the setting of recurrent UE-DVT with a central-venous catheter, $84 \%$ of physicians used anticoagulation, and half of them would keep the catheter in place (50.3\%). Of note, $7 \%$ would not use anticoagulation in this scenario; $47 \%$ of the participants would treat for 12 weeks, $26 \%$ for as long as the catheter was in place, $15 \%$ for less than 12 
weeks, and $9 \%$ used varied durations There was no statistical difference between specialties in the duration of anticoagulation utilized ( $p=0.25$ ) (Figure 3). The 2012 ACCP guidelines recommend that anticoagulation be continued for the time that the catheter was in place. ${ }^{10}$ Only $25 \%$ of the surgeons and $25 \%$ of the medical physicians followed that guideline. The recommended drugs in the guideline are LMWH, fondaparinux, or UFH. ${ }^{10}$ In our survey, the most common choice was DOACs ( $42 \%$ surgeons, vs. $37 \%$ medical specialists), followed by UFH or LMWH bridged to warfarin, (37\% surgeons, vs. $32 \%$ medical specialists). There was no difference in choice of drugs between surgeons and medical doctors, $p=0.69$ (Figure 4).

No specific recommendations exist for prophylaxis or follow-up of UE-DVT. A majority of participants would not use any prophylaxis; however, $14.2 \%$ would use low-dose anticoagulation, while $64 \%$ would follow patients with a venous duplex ultrasound, and $24 \%$ with physical exam alone.

We identified those physicians who reported treating higher volumes of UE-DVT patients ( $>3$ per month) and analyzed their responses separately. This included 35 participants (24\%) and their treatment choices differed from published guidelines. While the majority (64\%) would anticoagulate IVD-related UE-DVT, half of them would keep the IVD in place and the other half would remove it. For long-term IVDs associated with UE-DVT, 32\% would follow the guidelines and anticoagulate for as long as the device was in place, and $38 \%$ would anticoagulate only for 12 weeks. UFH or LMWH followed by warfarin was the preferred anticoagulant in $41 \%$ of participants, and $29 \%$ would use DOACS as first line of treatment.

Only $10 \%$ of participants believed that current guidelines were appropriate and based on strong evidence; a majority felt that there was a lack of adequate and convincing clinical evidence to guide treatment. Of note, after reviewing all the responses, only four physicians (2.8\%) reported following all the guidelines (two vascular medicine, one hematology and one interventional radiology physician).

\section{Discussion}

Our survey demonstrates high variability in treatment decisions for intravenous-devicerelated UE-DVT across multiple specialties. This included decisions on prophylaxis, need for therapeutic anticoagulation, type of anticoagulant, duration of treatment, approach to retaining or removing the device, and approach to follow-up. Only $10 \%$ of participants agreed with current guidelines. This was reflected in the even smaller proportion of physicians (2.8\%) actually following all currently available guidelines. Our survey targeted physicians expected to encounter patients with UE-DVT as part of their practice. The mean time in practice was 11.7 years and a majority of respondents treated one-to-two such patients every month. Results from our survey likely reflect practice patterns amongst physicians that are well-informed and experienced in this field.

Intravenous-devices, including central venous catheters are the most frequent risk factor for UE-DVT. ${ }^{7}$ In the absence of high-quality data, there is controversy on whether these patients 
require treatment and whether it is preferable to remove the device. The incidence of pulmonary embolism (PE) after UE-DVT is about $2 \%-2.8 \% 8,9$ and there is limited prospective evidence that anticoagulation reduces the incidence of fatal or non-fatal PE. In our survey, surgeons were more likely to remove intravenous catheters than physicians in medical specialties. Possible reasons for this difference in approach may be the type of patients treated by these specialists; medical patients usually require long-term intravenous catheters for antibiotics or chemotherapy that they may be more reluctant to remove, while surgeons tend to encounter patients with shortterm perioperative central lines. Use of anticoagulation was also different between specialists; it may be attributable to an increased risk of post-op bleeding in surgical patients. Reticence to use anticoagulation may also be due to the increased incidence of fatal, major, and minor bleeding in patients with UE-DVT. 6,8

Anticoagulation as well as catheter removal in IVD-related UE-DVT have been associated with symptomatic improvement, ${ }^{6}$ even though anticoagulation has not been associated with faster thrombus res-olution. ${ }^{13}$ The pathophysiology of non-device-related UE-DVT is different from IVD-related UED-DVT, and they benefit from anticoagulation, sometimes thrombolysis, and in cases of thoracic outlet syndrome, a cervical rib resection. ${ }^{14}$ The evidence for anticoagulation in the prevention of acute PE and thrombus extension in patients with LE-DVT is clearly strong. ${ }^{10,11}$ These indirect experiences may explain why a majority $(70.1 \%)$ of participants in our survey reported using some form of anticoagulation in their patients. The ACCP guidelines (2012) for UE-DVT recommend UFH, LMWH or fondaparinux, followed by warfarin. ${ }^{15}$ The ACCP guidelines (2016) for LE-DVT recommend DOACS based on strong recent evi-dence. ${ }^{16-21}$ In the absence of large prospective studies, there is no guidance on the use of DOACS in UE-DVT. ${ }^{11}$ One small prospective study in cancer patients with UE-DVT shows increased catheter patency though higher bleeding complications (13\%) with rivaroxaban. ${ }^{21}$ There is one ongoing study evaluating rivaroxaban for catheter-associated UE-DVT (), and two ongoing studies evaluating apixaban in similar patients ( and ). It is reasonable to consider initiating anticoagulation using an agent with a short half-life allowing quick reversal for bleeding in critically ill patients. The current absence of data to make an informed decision, however, explains why there was significant variability in the choices made by our participants (39.2\% used DOACS, others used UFH or LMWH).

The incidence of UE-DVT is $2.2 \%{ }^{2}$ in shortterm catheters and $6 \%$ in long-term catheters. ${ }^{3}$ Observational studies suggest that prophylactic low-dose warfarin may prevent UE-DVT. 4,5,22 This must be weighed against potential bleeding complications. Without definitive data, it is understandable that few participants (14.2\%) used anticoagulation for prophylaxis. UE-DVT associated with long-term catheters left in place has increased risks of recurrent thrombosis and upper extremity dysfunction. ${ }^{22,23}$ Low-dose warfarin or LMWH prophylaxis, however, reduced catheter-related UE-DVT4. Although the ACCP guidelines (2012) recommend continued anticoagulation for UE-DVT for the duration that the device is in place, only $26 \%$ of participants elected to follow this recommendation. There are no data to guide recommendations on the need or benefit of follow-up after an UE-DVT, or the optimal imaging modality to be used for follow-up. It is not known whether this would alter management decisions in the long term, such as determination of the intensity or 
continuation of anticoagulation. In our survey, $64 \%$ of physicians reported following their patients with a venous duplex ultrasound.

\section{Limitations}

We do not have a pre-survey test of participants to prove their knowledge about current treatment guidelines for UE-DVT. We did, however, ask about the number of patients with UE-DVT that they treated and the number of years they had been in practice as surrogates for experience and knowledge. The response rate of $8 \%$ was low. The emails were obtained from a hospital and other databases and it is not clear whether they were up to date. There was no incentive provided to complete the survey. Finally, it is possible that institutional firewalls blocked many of our communications. Hence, the proportion of responders may be artificially low since our survey may not have reached a large number of participants.

\section{Conclusion}

There is great variability in the management of IVD-related UE-DVT across specialties. This is primarily driven by a lack of high-quality prospective data informing these decisions. As a result, currently available guidelines, derived largely from imperfect data on UE-DVT or extrapolated from LE-DVT studies, are not being utilized and implemented. There is a clear need for large well-designed studies focused on determining optimal prophylaxis, treatment and follow-up for IVD-related UE-DVT.

\section{Funding}

The author(s) disclosed receipt of the following financial support for the research, authorship, and/or publication of this article: VA Merit awards RX000995 and CX001621, and NIH awards NS080168, NS097876 and AG000513 (BKL); NIH awards AG028747, DK072488, and Baltimore VA Medical Center GRECC (JS and TM).

\section{References}

1. O'Grady NP, Alexander M, Burns LA, et al. Guidelines for the prevention of intravascular catheterrelated infections. Clin Infect Dis 2011; 52: e162-e193. [PubMed: 21460264]

2. Lamontagne F, McIntyre L, Dodek P, et al. Nonleg venous thrombosis in critically ill adults. JAMA Intern Med 2014; 174: 689. [PubMed: 24638843]

3. Young AM, Billingham LJ, Begum G, et al. Warfarin thromboprophylaxis in cancer patients with central venous catheters (WARP): an open-label randomised trial. Lancet 2009; 373: 567-574. [PubMed: 19217991]

4. Lavau-Denes S, Lacroix P, Maubon A, et al. Prophylaxis of catheter-related deep vein thrombosis in cancer patients with low-dose warfarin, low molecular weight heparin, or control: a randomized, controlled, phase III study. Cancer Chemother Pharmacol 2013; 72: 65-73. [PubMed: 23636449]

5. Kovacs MJ, Kahn SR, Rodger M, et al. A pilot study of central venous catheter survival in cancer patients using low-molecular-weight heparin (dalteparin) and warfarin without catheter removal for the treatment of upper extremity deep vein thrombosis (The Catheter Study). J Thromb Haemost 2007; 5: 1650-1653. [PubMed: 17488349]

6. Malinoski DJ, Ewing T, Patel MS, et al. The natural history of upper extremity deep venous thromboses in critically ill surgical and trauma patients: what is the role of anticoagulation? J Trauma 2011; 71: 312-316. [PubMed: 21825932]

7. Joffe HV, Kucher N, Tapson VF, et al. Upper-extremity deep vein thrombosis: a prospective registry of 592 patients. Circulation 2004; 110: 1605-1611. [PubMed: 15353493] 
8. Baumann Kreuziger L, Onwuemene O, Kolesar E, et al. Systematic review of anticoagulant treatment of catheter-related thrombosis. Thromb Res 2015; 136: 1103-1109. [PubMed: 26342400]

9. Levy MM, Albuquerque F and Pfeifer JD. Low incidence of pulmonary embolism associated with upper-extremity deep venous thrombosis. Ann Vasc Surg 2012; 26: 964-972. [PubMed: 22749742]

10. Kearon C, Akl EA, Comerota AJ, et al. Antithrombotic therapy for VTE disease: antithrombotic therapy and prevention of thrombosis, 9th ed: American College of Chest Physicians evidencebased clinical practice guidelines. Chest 2012; 141(2 Suppl.): e419S-e496S. [PubMed: 22315268]

11. Kearon C, Akl EA, Ornelas J, et al. Antithrombotic therapy for VTE disease: CHEST guideline and expert panel report. Chest 2016; 149: 315-352. [PubMed: 26867832]

12. Artino Artino AR, Durning SJ and Sklar DP. Guidelines for reporting survey-based research submitted to academic medicine. Acad Med 2018; 93: 337-340. [PubMed: 29485492]

13. Jones MA, Lee DY, Segall JA, et al. Characterizing resolution of catheter-associated upper extremity deep venous thrombosis. J Vasc Surg 2010; 51: 108-113. [PubMed: 19879094]

14. Kucher N. Deep-vein thrombosis of the upper extremities. N Engl J Med 2011; 364: 861-869. [PubMed: 21366477]

15. Kearon C, Akl EA, Comerota AJ, et al. Antithrombotic therapy for VTE disease. Chest 2012; 141: e419S-e496S. [PubMed: 22315268]

16. Schulman S, Kearon C, Kakkar AK, et al. Dabigatran versus warfarin in the treatment of acute venous thromboembolism. N Engl J Med 2009; 361: 2342-2352. [PubMed: 19966341]

17. Bauersachs R, Berkowitz SD, Benjamin B, et al. Oral rivaroxaban for symptomatic venous thromboembolism (EINSTEIN). N Engl J Med 2010; 363: 1-12. [PubMed: 20573919]

18. Kuuskne $M$ and Dankoff J. Oral rivaroxaban for the treatment of symptomatic pulmonary embolism. N Engl J Med 2013; 15: 1-3.

19. Agnelli G, Buller HR, Cohen A, et al. Oral apixaban for the treatment of acute venous thromboembolism. N Engl J Med 2013; 369: 799-808. [PubMed: 23808982]

20. Agnelli G, Buller HR, Cohen A, et al. Apixaban for extended treatment of venous thromboembolism. N Engl J Med 2013; 368: 699-708. [PubMed: 23216615]

21. Davies GA, Lazo-Langner A, Gandara E, et al. A prospective study of Rivaroxaban for central venous catheter associated upper extremity deep vein thrombosis in cancer patients (Catheter 2). Thromb Res 2018; 162: 88-92. [PubMed: 28416213]

22. Saber W, Moua T, Williams EC, et al. Risk factors for catheter-related thrombosis (CRT) in cancer patients: a patient-level data (IPD) meta-analysis of clinical trials and prospective studies. $\mathrm{J}$ Thromb Haemost 2011; 9: 312-319. [PubMed: 21040443]

23. Prandoni P, Noventa F, Lensing AWA, et al. Post-thrombotic syndrome and the risk of subsequent recurrent thromboembolism. Thromb Res 2016; 141: 91-92. [PubMed: 26986754] 


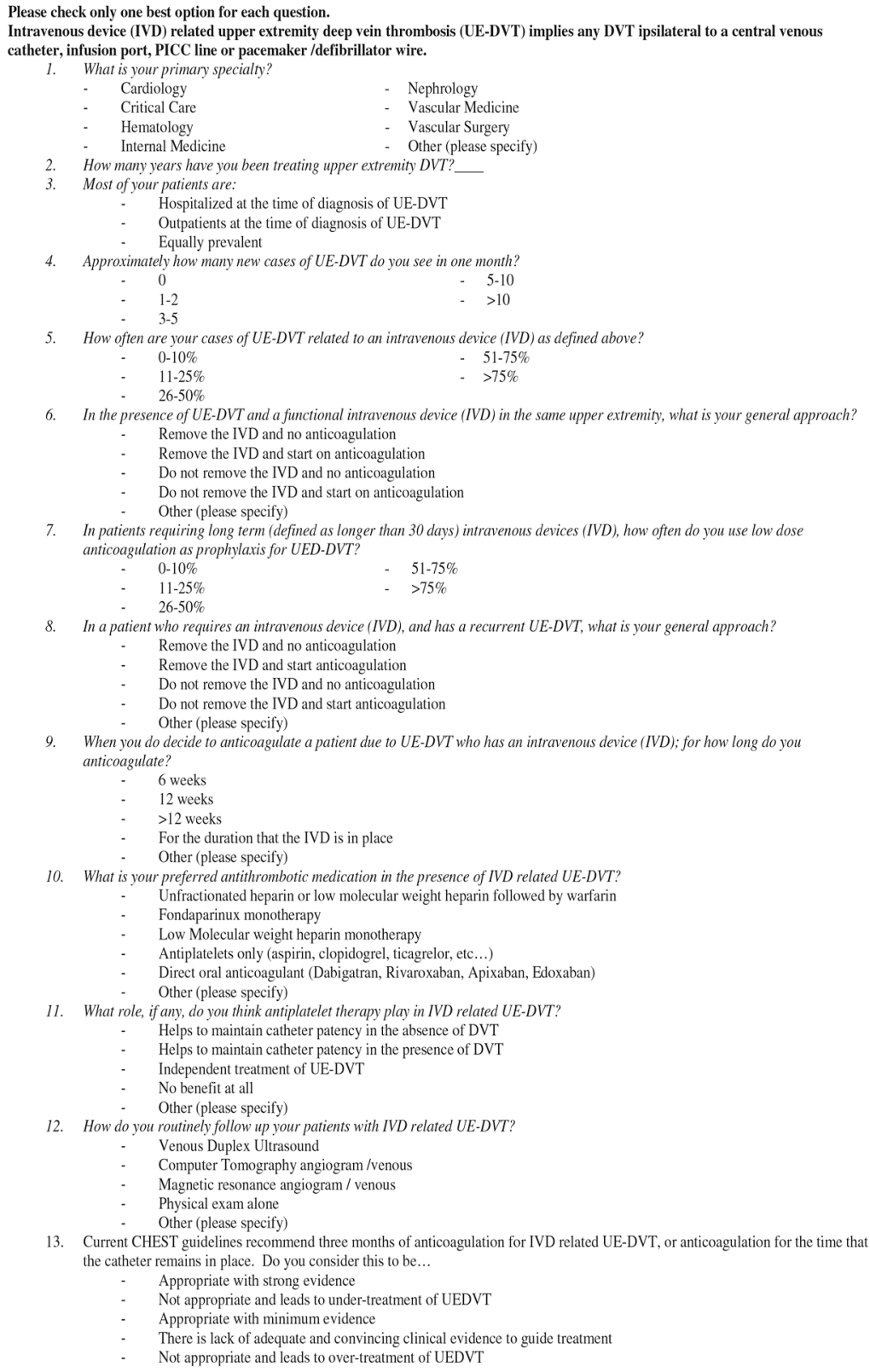

Figure 1.

Questionnaire for upper extremity deep vein thrombosis survey.

Phlebology. Author manuscript; available in PMC 2020 March 01. 


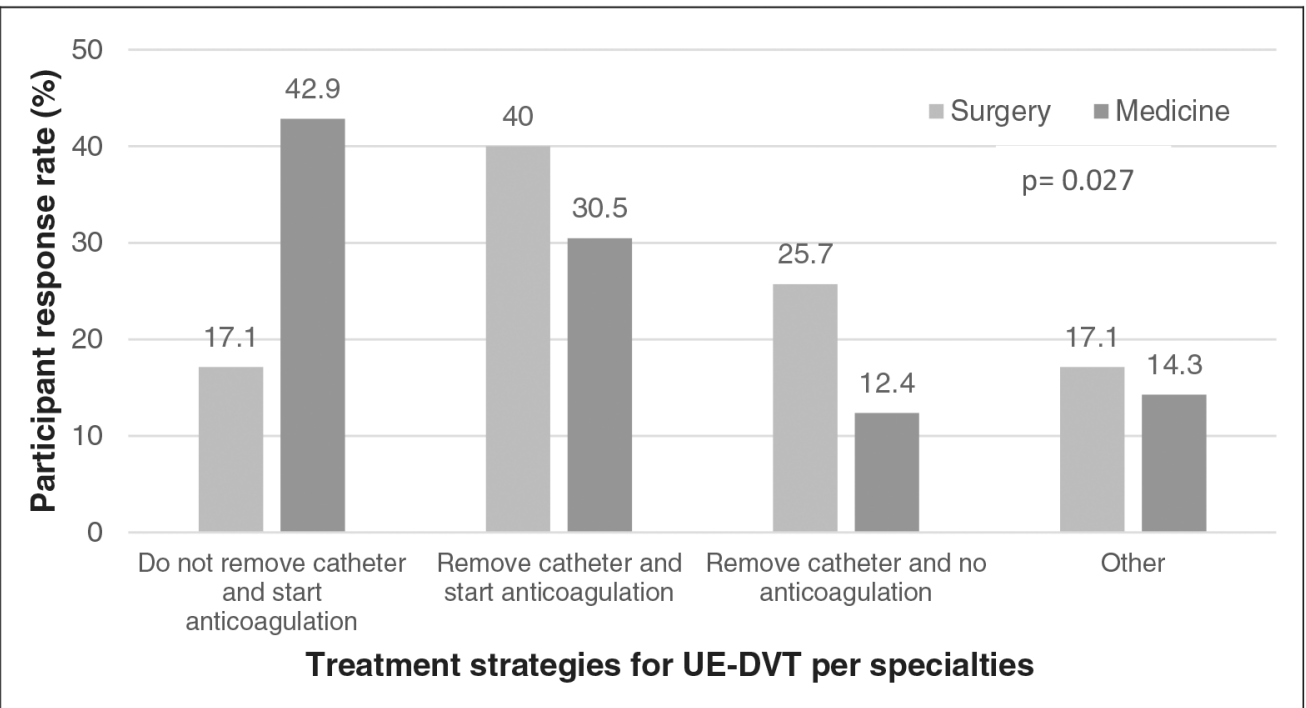

Figure 2.

Preferred treatment strategy by specialty Surgery vs. Medicine. 


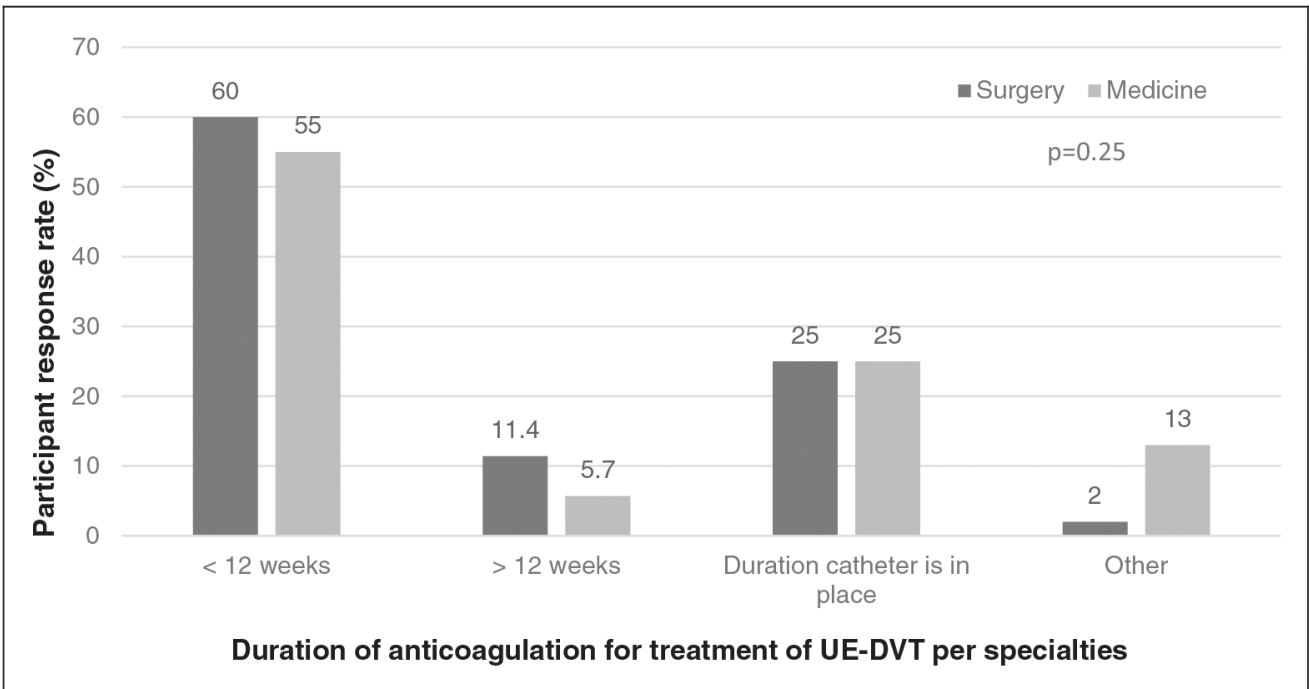

Figure 3.

Duration of anticoagulation in patients with functional long-term intravenous catheters in the presence of upper extremity deep vein thrombosis (UE-DVT) by specialty Surgery vs.

Medicine. 


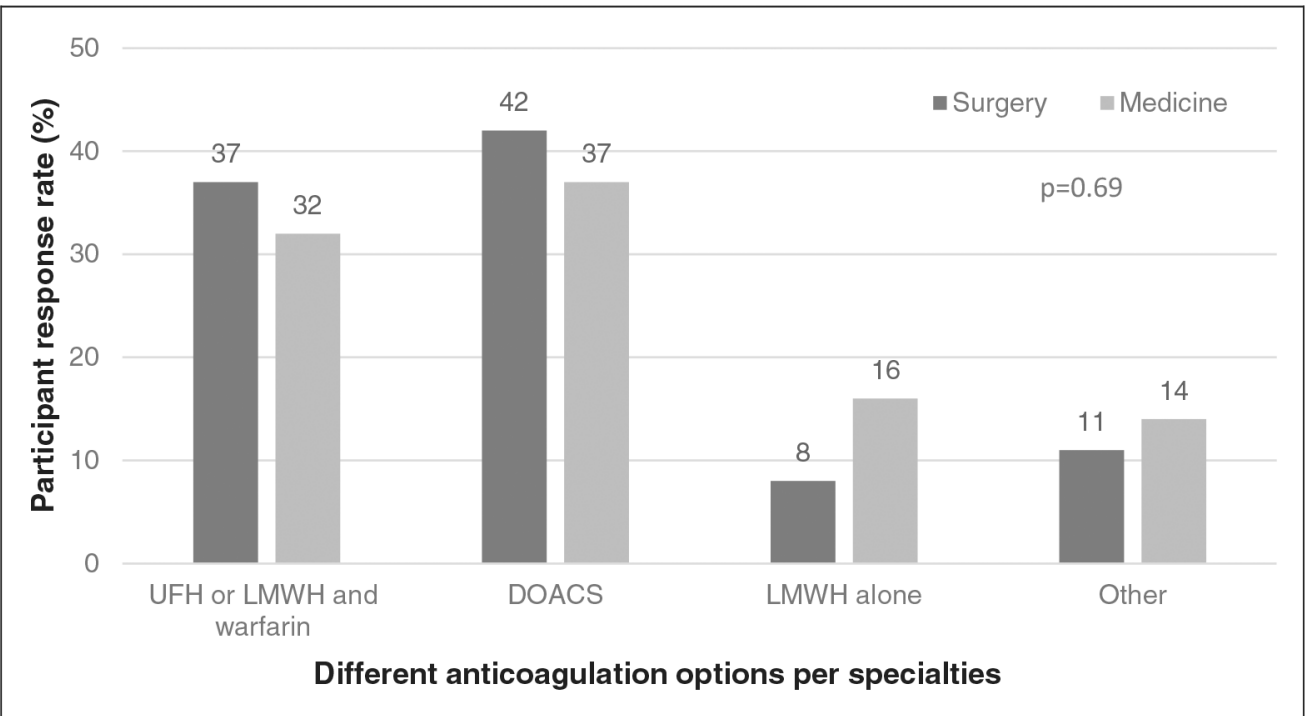

Figure 4.

Variability in preferred type of anticoagulation for patients with upper extremity deep vein thrombosis per specialty Surgery vs. Medicine. UFH: unfractionated heparin; LMWH: lowmolecular weight heparin; DOACS: direct oral anticoagulants. 